\section{REBALANCEAMENTO DO SISTEMA DE BICICLETAS COMPARTILHADAS: EXPERIMENTO REALIZADO NA CIDADE DO RIO DE JANEIRO}

\author{
Felipe Pereira Rosa Pierrotti \\ Instituto Militar de Engenharia \\ Praça Gen. Tibúrcio, 80 - Urca, Rio de Janeiro - RJ \\ pierrotti.felipe@gmail.com \\ Orivalde Soares da Silva Júnior \\ Instituto Militar de Engenharia \\ Praça Gen. Tibúrcio, 80 - Urca, Rio de Janeiro - RJ \\ orivalde@yahoo.com.br
}

\begin{abstract}
RESUMO
Com intuito de minimizar o custo do transporte de bicicletas compartilhadas para balanceamento das estações de retirada e entrega de bicicletas na cidade do Rio de Janeiro foi desenvolvido um modelo matemático para o problema e aplicado no software AIMMS. Foram analisados dados históricos e utilizado como base o problema do caixeiro viajante associado ao problema da mochila. A análise resultou na determinação do caminho mínimo que atende as demandas das diferentes estações e respeita a capacidade máxima dos veículos transportadores de bicicletas.
\end{abstract}

Palavra-chave: Rebalanceamento; Otimização; Sistema de Bicicleta Compartilhada.

\begin{abstract}
In order to minimize the transportation cost of bike sharing rebalance in Rio de Janeiro a mathematic model was developed and applied in the software AIMMS. A historical data was use as the basis of the Traveler Salesman Problem associated with the Knapsack Problem. The regression analysis of the minimum path is one of the requirements of the different stations and is a concern of the bicycle transporters.
\end{abstract}

Keywords: Rebalance; Bike-sharing system (BSS); Management.

\section{Como Citar:}

PIERROTTI, Felipe Pereira Rosa; SILVA JÚNIOR, Orivalde Soares da. Rebalanceamento do Sistema de Bicicletas Compartilhadas: Experimento Realizado na Cidade do Rio de Janeiro. In: SIMPÓSIO DE PESQUISA OPERACIONAL E LOGÍSTICA DA MARINHA, 19., 2019, Rio de Janeiro, RJ. Anais [...]. Rio de Janeiro: Centro de Análises de Sistemas Navais, 2019. 


\section{INTRODUÇÃO}

O Sistema de Bicicletas Compartilhadas é uma ferramenta de complementação e ramificação do sistema de transporte público, que cresce nas grandes cidades de todo o mundo. Veículos compartilhados se apresentam como a mais recente e inovadora solução de mobilidade urbana que alia a sustentabilidade e baixo custo com praticidade e conveniência de permitir que a viagem de volta seja feita por outro modal, o que não é possível em veículos próprios.

Pesquisas recentes apontam que 60\% dos trajetos feitos por veículo próprio, na cidade de São Paulo, percorrem uma distância inferior à $5 \mathrm{~km}$. Ou seja, os modelos de mobilidade urbana para pequenas e médias distâncias, como as bicicletas compartilhadas podem substituir os carros próprios gerando maior qualidade de vida ao cidadão e diminuindo a concentração de carros nas vias, no âmbito social. O sistema de bicicletas compartilhadas (SBC) (em inglês Bike Sharing Systems - BSS), é encontrado nas principais cidades por todo mundo como Munique, Barcelona, Londres, Xangai e Amsterdã. O baixo desempenho dos transportes coletivos urbanos, o uso e ocupação do solo e, a cada vez maior, preocupação com o meio ambiente e a qualidade do ar, fortalecem a utilização de meios de transporte não emissores de gases.

O sistema de bicicleta compartilhada se caracteriza pela possibilidade ao usuário de recolha e devolução da bicicleta em qualquer estação da cidade em que se tenha bicicleta ou vaga disponível, respectivamente. Foi verificado que, na cidade do Rio de Janeiro, a incidência da indisponibilidade de bicicletas ou a falta de vagas para devolução é grande, o que ocasiona grande descontentamento aos usuários e possibilidade de perda dos clientes. Estes pontos críticos se justificam pela variação de demanda horária e diária em cada estação, exemplificada pelo movimento pendular diário exercido por milhões de pessoas diariamente que se deslocam em direção ao seu trabalho ou local de estudo pela manhã, e ao fim do dia se locomovem em sentido oposto, com o objetivo de retorno ao lar, o que gera o desequilíbrio da estação que por vezes falta de bicicletas para retirada dos usuários e por vezes os usuários que já usufruíram do serviço não conseguem finaliza-lo na estação desejada por falta de docks, como são chamados os locais onde as bicicletas são encaixadas quando devolvidas, disponíveis.

Para a solução deste problema são utilizados caminhões de pequeno porte, que transportam as bicicletas das estações que apresentam seu estoque próximo ao limite da capacidade para as estações que, no mesmo momento, estão com baixos níveis em seu estoque, buscando que todas as estações estejam próximas a um estoque adequado, que permita a retirada pelos usuários e permita que bicicletas sejam devolvidas ao fim do uso.

O artigo está organizado iniciando nesta seção de introdução, na seção 2 é apresentada a revisão da literatura sobre o problema de rebalanceamento de bicicletas, na seção 3 são apresentados o problema estudado e o modelo matemático proposto, na seção 4 são apresentados os resultados e discussões e finalmente, na seção 5 são apresentadas as conclusões.

\section{REVISÃO DE LITERATURA}

Reiss e Bogenberger (2016) definem o sistema público de bicicletas compartilhadas como uma opção progressiva para a mobilidade urbana, não só para os usuais utilizadores mas também aos turistas e usuários pontuais, no entanto, que o sistema é eficiente se as bicicletas estão disponíveis no momento que os usuários desejam. Foi utilizado um modelo baseado em dados de GPS do sistema compartilhado de bicicletas da cidade de Munique, Alemanha, construído um modelo de previsão de demanda dependente de fatores como 
estação do ano, condições meteorológicas e dias úteis ou fim de semana. Como conclusão foi possível verificar a mudança de demanda, que baixam drasticamente nas estações da primavera e outono e nos períodos de chuva, quando é preciso cerca de três horas para que seja atingido o nível médio de viagem. Foi verificado, também, que as zonas residências apresentam uma grande demanda no período matinal enquanto as zonas localizadas perto do centro da cidade exibem baixa demanda neste período e alta demanda no período noturno. Caggiani e Ottomanelli (2012) destacam como questão crucial para os sistemas de compartilhamento de bicicletas a distribuição desequilibrada no espaço e no tempo das bicicletas entre as estações. Foi proposto um sistema de suporte à decisão mais flexível para minimizar o custo de redistribuição determinando os padrões, velocidades e intervalos de reposicionamento de fluxos. O método de otimização foi aplicado a um sistema simulado, baseado nos dados do sistema de bicicletas compartilhadas da cidade de Barcelona, na Espanha, que pode ser considerado como um módulo de um sistema de bicicletas compartilhadas real, porém mais amplo graças à arquitetura escalável do sistema de suporte à decisão. Magalhães et al. (2018) elencam os fatores determinantes para a geração de viagens por bicicletas, como disponibilidade de infraestrutura para circulação de bicicletas, estacionamento, a temperatura máxima na região e número de acidentes e número de interseções, proximidade a vias com estrutura e número de faixas paras os trechos onde não há infraestrutura para circulação de bicicletas. O estudo feito na cidade do Rio de Janeiro identificou um modelo direto de demanda de viagens com infraestrutura necessária para circulação de ciclistas.

\section{PROBLEMA E METODOLOGIA}

Este artigo considera o Sistema de Bicicletas Compartilhadas como um Problema do Caixeiro Viajante respeitando a capacidade do veículo que transporta. Foram coletados dados através do acompanhamento em tempo real das estações, após análise foi possível determinar, através da mediana, valor que mais se repete na amostra, a demanda mais provável para o período determinado. O período escolhido para o balanceamento foi o período compreendido entre as 08:00 às 12:00, para que se estabilize a estação e ao decorrer do dia se evite momentos críticos.

Este artigo busca solucionar um problema recorrente nas estações de bicicletas compartilhadas em todo o mundo, decorrente da variação de demanda durante o decorrer do dia por bicicletas. A variação da solicitação de bicicletas gera, por consequência, a variação da disponibilidade das bicicletas a serem retiradas nas estações, seja pelo excesso de bicicletas disponíveis, impedindo que demais bicicletas sejam devolvidas por usuários que almejam finalizar a utilização do produto, seja pela carência de bicicletas disponíveis, que impede aos usuários de iniciarem sua jornada utilizando as bicicletas.

\subsection{OBTENÇÃO DOS DADOS}

Foram obtidos dados a partir do acompanhamento entre o período de 27 de março à 15 de abril de 2019 de 81 (oitenta e uma) estações espalhadas pela cidade do Rio de Janeiro, com um intervalo de amostragem de 5 (cinco) minutos, onde continham a estação, a quantidade de bicicletas e vagas disponíveis. Através destes dados foi elaborada uma análise do comportamento característico de cada estação através de comparação entre amostras. A partir desta etapa foram escolhidas 15 (quinze) estações de comportamento complementar para verificação da metodologia. 


\subsection{OTIMIZAÇÃO DO REBALANCEAMENTO}

O modelo proposto para realizar a otimização do transporte de bicicletas entre as estações que não estão devidamente balanceadas é apresentado pelo modelo matemático, o qual é representado pelas expressões de (1) à (9).

$\mathrm{O}$ conjunto $\mathrm{E}=\left\{e_{1}, e_{2}, \ldots, e_{n}\right\}$ representa as estações de bicicletas, o conjunto $\mathrm{A}=\left\{a_{i j} \cdot e_{i}\right.$ ,$\left.e_{j} \in \mathrm{E}\right\}$ representa os arcos de cada estação $i$ para estação $j$, com $i \neq j$, os parâmetros $D_{i j}$ representam as distância entre as estações $i$ e $j$, os parâmetros Dem $_{i}$ representam as demandas de bicicletas a serem coletadas ou entregues das estações $i$ (uma demanda negativa indica entrega e uma demanda positiva indica coleta), o parâmetro Cap indica a capacidade máximo do veículo em bicicletas, o parâmetro Bikes representa a quantidade carga inicial de bicicletas do veículo, as variáveis de decisão $Y_{i j}$ representam a quantidade de bicicletas transportadas da estação $i$ para a estação $j$ e as variáveis de decisão $x_{i j}$ indicam a sequencia de estações visitadas pelo veículos. A distância entre as estações foi considerada a distância euclidiana, calculada pela expressão (1).

$$
D_{i j}=\sqrt{\left(C O_{x i}-C O_{x j}\right)^{2}+\left(C O_{y i}-C O_{y j}\right)^{2}}
$$

Sendo, $C O_{x i}$ a longitude da estação $i, C O_{x j}$ a longitude da estação $j, C O_{y i}$ a latitude da estação $i$ e $C O_{y j}$ a latitude da estação $j$. As variáveis de decisão $x_{i j}$ assumem apenas valores iquais a 0 ou 1 , conforme ilustra a expressão (2).

$$
\mathrm{x} \mathrm{ij}=\left\{\begin{array}{c}
1, \text { se a arco ij foi selecionado para a rota } \\
0, \text { caso contrário }
\end{array}\right\}
$$

As variáveis de decisão relacionadas à quantidade de bicicletas transportadas são representadas por $Y_{i j}$, sendo a quantidade de bicicletas transportadas da estação $i$ para a estação $j$.

$$
\operatorname{Min} z=\sum_{(i, j) \in A} D_{i j} * x_{i j}
$$

s.a.

$$
\begin{gathered}
\sum_{j \in E} x_{i j}=1 \forall i \in E \\
\sum_{i \in E}^{i \in E} x_{i j}=1 \forall j \in E \\
\sum_{(i, j) \in A} Y_{i j} \leq C a p \\
\operatorname{Dem}_{i}=\sum_{j \in E} Y_{j i}-\sum_{j \in E} Y_{i j} \forall i \in E \\
\sum_{j \in E} Y_{1 j}=B i k e s \\
\sum_{(i, j) \in S} x_{i j} \leq|S|-1 \forall S \subset E \\
Y_{i j} \geq 0 \forall i, j \in E \\
x_{i j} \in\{0,1\} \forall i, j \in E
\end{gathered}
$$

A função objetivo (3) busca minimizar a distância total percorrida pelo veículo. As restrições (4) garantem que o veículo saia de cada estação apenas uma vez. As restrições (5) 
garantem que cada estação é visitada apenas uma vez pelo veículo. As restrições (6) garantem que a capacidade do veículo transportador seja respeitada. As restrições (7) indicam que a demanda de cada estação seja a quantidade de bicicleta que chega, com o veículo, à estação subtraído da quantidade que sai com o veículo da mesma estação. As restrições (8) realizam indicam que o veículo sai da estação inicial para realizar a coleta e entrega de bicicletas carregado com Bikes bicicletas. A estação inicial arbitrada foi a Central do Brasil. Uma dificuldade inerente ao Problema do Caixeiro Viajante é a criação de subrotas na solução ótima, que separariam em setores com rotas fechadas e independentes, sendo assim, as restrições (9) evitar que subrotas sejam criadas na solução, sendo $S$ o conjunto de subrotas das estações. As restrições (10) garantem a não negatividade das variáveis de decisão da quantidade de bicicletas transportadas de $i$ para $j$ e as restrições (11) garantem que as variáveis de decisão da rota assuma apenas valores binários, 0 ou 1.

A Figura 1 mostra um exemplo da criação de sub rotas, onde a solução ótima encontrada traça duas rotas em separado. Por ordem do objetivo deste trabalho ser a definição de uma única rota, a restrição garante que a solução ótima tenha o aspecto demonstrado pela Figura 2.

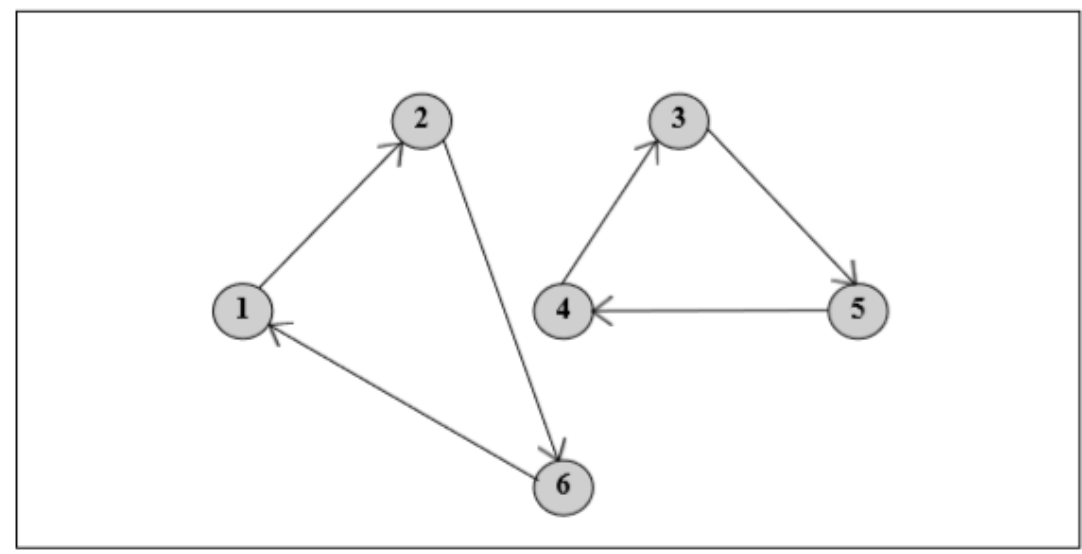

Figura 1 - Subrotas. Fonte: Alves, Delgado, 1997

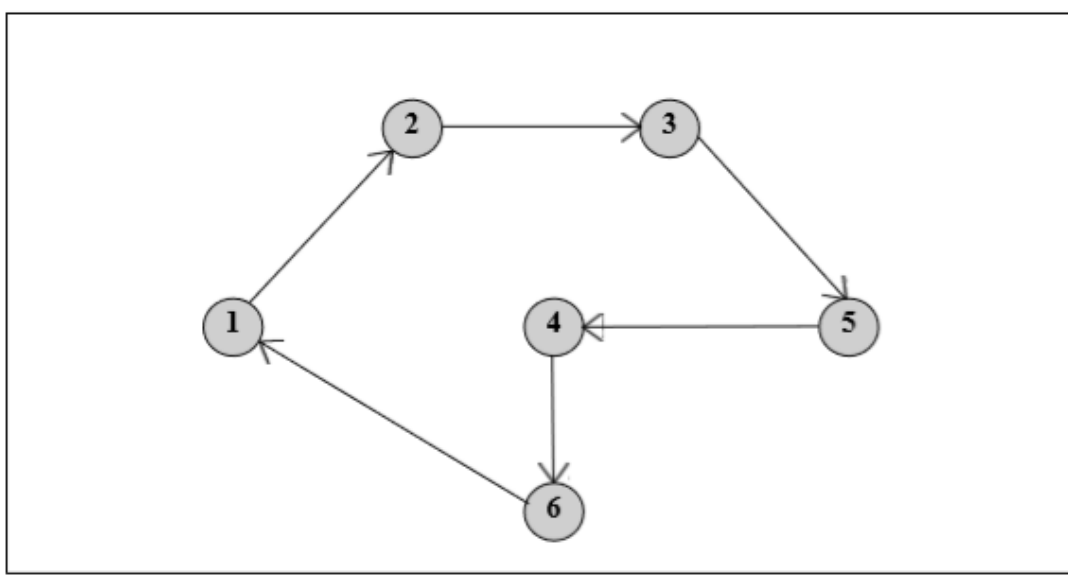

Figura 2 - Eliminação das Subrotas. Fonte: Alves, Delgado, 1997

\section{RESULTADOS E DISCUSSÕES}

O Sistema de bicicletas compartilhadas da cidade do Rio de Janeiro, gerenciado pela empresa Tembici, possui 260 estações espalhadas pela cidade e 2600 bicicletas em 
circulação. A cidade do Rio de Janeiro é a campeã brasileira neste tipo de mobilidade urbana sustentável. Contudo o problema de desbalanceamento das estações ainda faz com que muitos usuários não possam utiliza-las, conforme mostrado na Figura 3, devido a ausência de bicicletas para retirada.

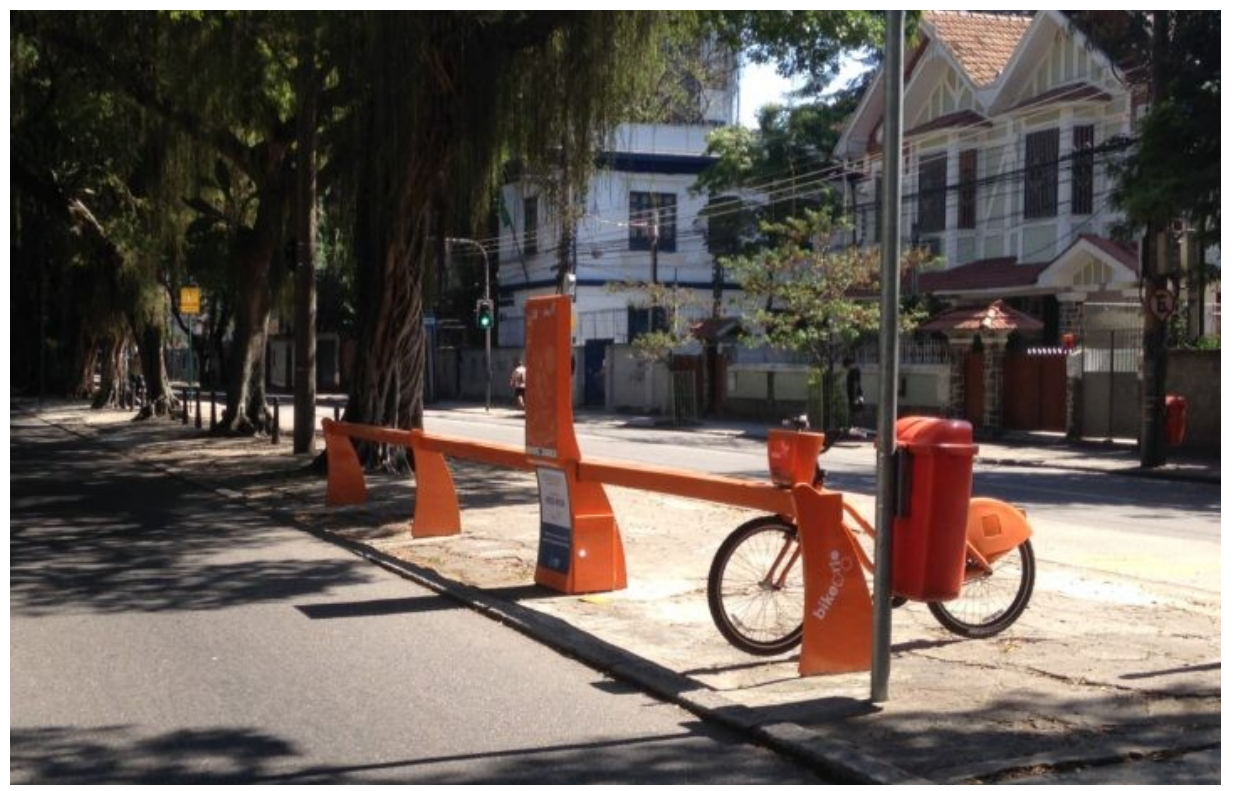

Figura 3 - Estação Av. Pasteur sem bicicletas

Em outro período do mesmo dia, a mesma estação pode causar aborrecimento aos usuários, no entanto, de outra forma, ao se deparar com a estação completa, como visto na Figura 4, sem a possibilidade de se devolver a bicicleta, conforme necessário para finalização da viagem.

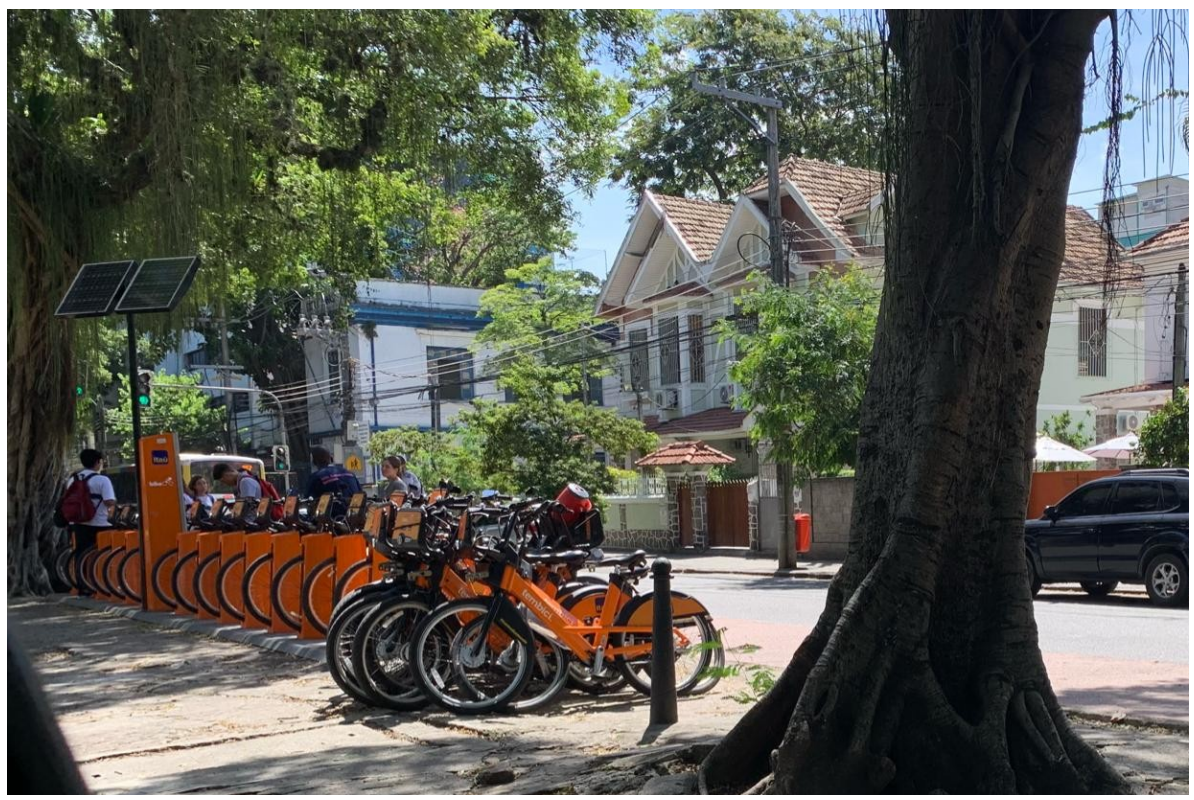

Figura 4 - Estação Av. Pasteur com capacidade esgotada

O modelo de veículo executor do remanejamento entre estações foi considerado com a capacidade de 10 bicicletas, sendo o menor veículo da frota utilizada pela empresa para este serviço. Foi considerada, também, por simplificação, uma demanda fixa, fruto da 
mediana dos dias amostrados, para cada estação. O período considerado para o rebalanceamento foi o turno da manhã, para que seja possível prevenir, no decorrer do dia, novos desbalanceamentos.

O modelo matemático foi executado no AIMMS, software de modelagem e otimização que gerou a rota e os gráficos apresentados a seguir. Estas informações foram analisadas com auxílio do software Excel para que o resultado fosse melhor interpretado. A rota gerada pelo AIMMS é apresentada pela figura a seguir, considera-se o início na estação Central do Brasil, e com o caminhão, de capacidade de até 28 bicicletas, carregado com 10 bicicletas para que possa atender a demanda da primeira estação. A rota traçada pelo AIMMS considerou a distância linear entre as estações, conforme visto na Figura 5.

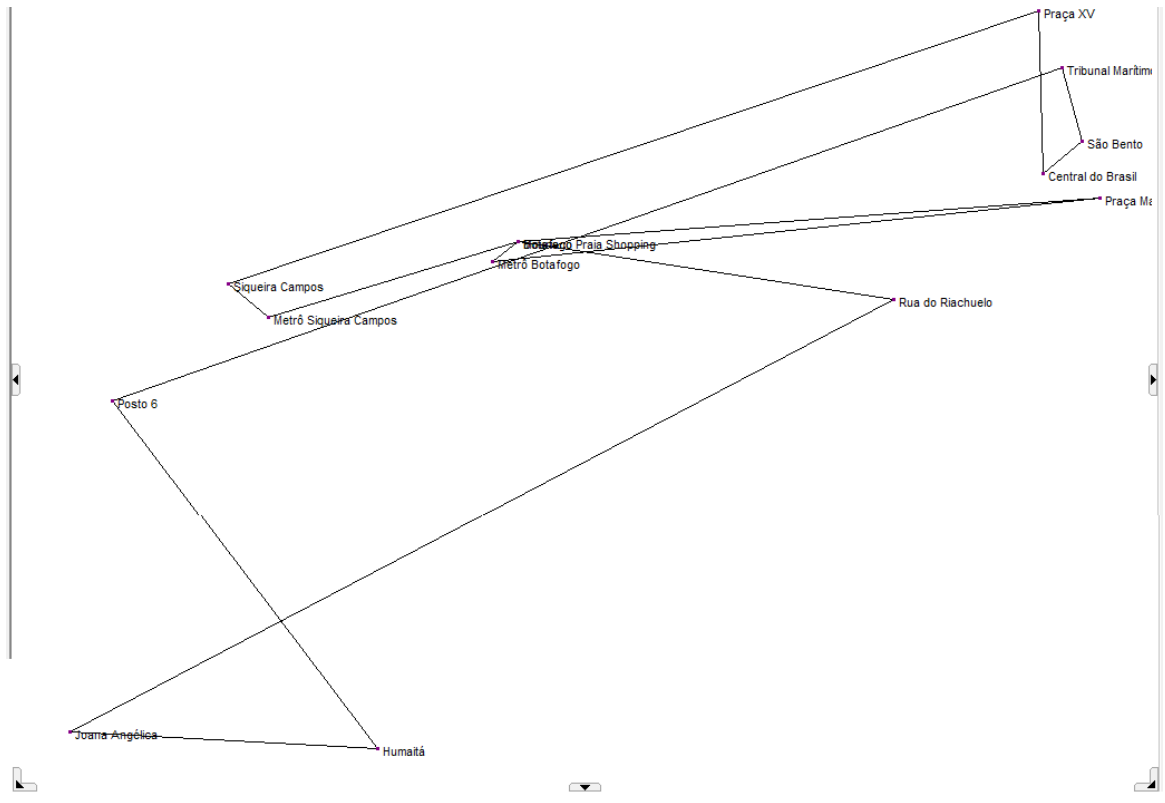

Figura 5 - Roteiro de coletas e entregas de bicicletas representado no AIMMS

A Figura 6 mostra, com a utilização da ferramenta Google Maps, o roteiro, resultado da simulação executada no software AIMMS, entre as diversas estações para realização do balanceamento.

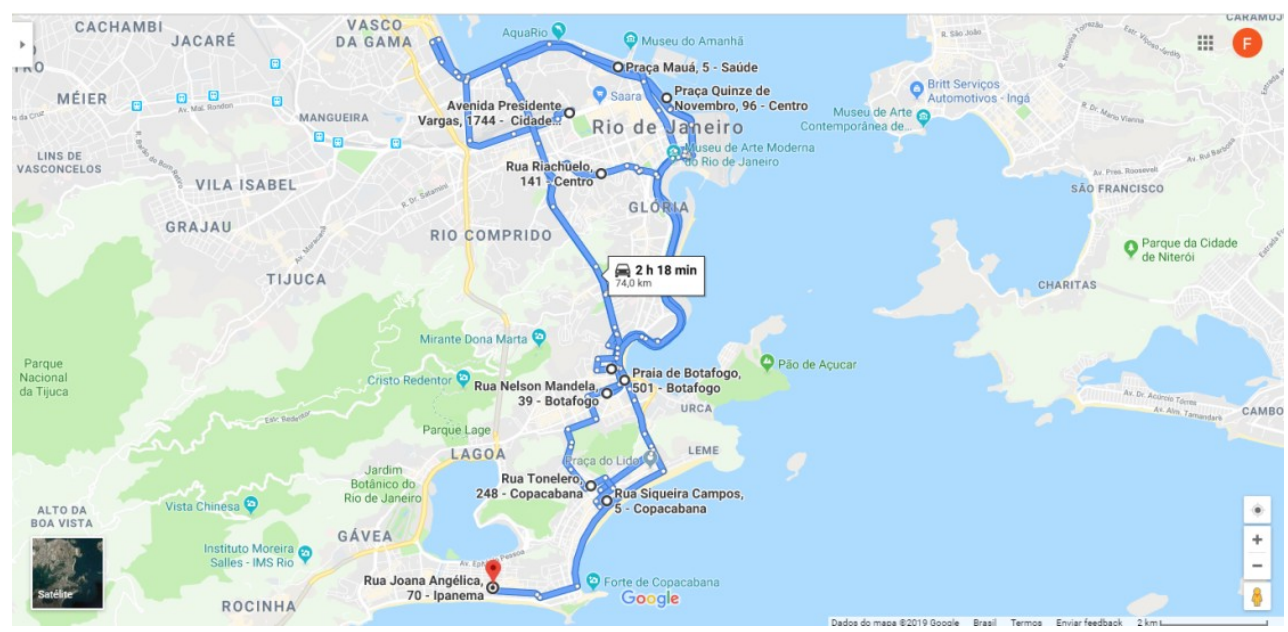

Figura 6 - Rota de balanceamento. Fonte: Google

A Tabela 1 mostra a estação de saída (i) e a de destino (j), a demanda calculada para a estação de destino (j), quantas bicicletas o caminhão de transporte apresenta antes e 
quantas bicicletas há no caminhão após o carregamento da estação de destino (j). As seguintes colunas são apresentadas: Saída (i) - Estação de partida do veículo de transporte em direção à estação $j$; Chegada (j) - Estação de chegada do veículo de transporte proveniente da estação $i$; Demanda Estação $j$ - Quantidade de bicicletas na estação $j$ necessárias para alcançar o equilíbrio; Qt inicial - Quantidade de bicicletas transportadas pelo caminhão ao chegar na estação $j$; Qt final - Quantidade de bicicletas presentes no caminhão de transporte ao sair da estação $j$.

Tabela 1 - Rota, Demanda e Quantidade transportada

\begin{tabular}{c|c|c|c|c}
\hline Saída(i) & Destino(j) & Demanda Estação j & $\begin{array}{c}\text { Qt inici- } \\
\text { al }\end{array}$ & Qt final \\
\hline Central do Brasil & Praça XV & 5 & 10 & 5 \\
\hline Praça XV & Siqueira Campos & -4 & 5 & 9 \\
\hline Siqueira Campos & Metrô Siqueira Campos & -2 & 9 & 11 \\
\hline Metrô Siqueira Campos & Mourisco & -3 & 11 & 14 \\
\hline Mourisco & Metrô Botafogo & -5 & 14 & 19 \\
\hline Metrô Botafogo & Praça Mauá & -1 & 19 & 20 \\
\hline Praça Mauá & Botafogo Praia Shopping & 5 & 20 & 15 \\
\hline Botafogo Praia Shopping & Rua do Riachuelo & 5 & 15 & 10 \\
\hline Rua do Riachuelo & Joana Angélica & 1 & 10 & 9 \\
\hline Joana Angélica & Humaitá & -2 & 9 & 11 \\
\hline Humaitá & Posto 6 & -5 & 11 & 16 \\
\hline Posto 6 & Tribunal Marítimo & -3 & 16 & 19 \\
\hline Tribunal Marítimo & São Bento & 3 & 19 & 16 \\
\hline São Bento & Central do Brasil & 5 & 16 & 11 \\
\hline
\end{tabular}

\section{CONCLUSÕES}

As séries históricas, obtidas através da amostragem, auxiliaram a compreender o comportamento característico de cada estação, através do modelo matemático foi gerado o roteiro passando por todas as estações, respeitando as condições e restrições impostas pelo modelo, atendendo, desta forma, o propósito e objetivo do trabalho.

Desta forma, o balanceamento das estações baseado nas séries históricas permite aos operadores planejar a rota antecipadamente e prevenir os momentos críticos evitando que usuários tenham a experiência negativa de encontrar estações impossibilitadas de atender aos seus objetivos.

O modelo matemático, utilizado na aplicação de um pequeno estudo de caso, com parte das estações do sistema de compartilhamento de bicicletas do Rio de Janeiro, com base no modelo do Problema do Caixeiro Viajante Capacitado, por ter imposta a limitação do veículo transportador das bicicletas, atendeu ao trabalho proposto gerando resultados que permitiram a validação do modelo. 


\section{REFERÊNCIAS BIBLIOGRÁFICAS}

[1] SILVA, Rodolfo Celestino dos Santos. Rebalanceamento dinâmico de sistema de bicicletas compartilhadas e aplicação de simulação com otimização a um sistema brasileiro. 2018. Tese (Doutorado) - Escola Politécnica da Universidade São Paulo, [S. l.], 2018.

[2] REISS, S.; BOGENBERGER, K. A Relocation Strategy for Munich's Bike Sharing System: Combining an operator-basedand a user-based Scheme. 19th EURO Working Group on Transportation Meeting, Istanbul, Turkey, 2016.

[3] ALVES, R; DELGADO, C. Programação Linear Inteira, Faculdade de Economia da Universidade do Porto, Portugal, 1997.

[4] CAGGIANI, Leonardo; OTTOMANELLI, Michele. A modular soft computing based method for vehicles repositioning in bike-sharing systems. EURO Working Group on Transportation: EWGT 2012, Bari, Itália, 2012. DOI 10.1016/j.sbspro.2012.09.785.

[5] MAGALHÃES, Jefferson Ramon Lima; CAMPOS, Vânia Barcellos Gouvêa; BANDEIRA, Renata Albergaria de Mello. Análise de variáveis para esmava de viagens por bicicletas: Um estudo no município do Rio de Janeiro, Brasil. Revista Transportes, [S. l.], Dezembro 2018. DOI DOI:10.14295/transportes.v26i4.1392. Disponível em: https://www.revistatransportes.org.br/anpet/article/view/1392. 